\title{
MULTI-PLAYER DIFFUSION GAMES ON GRAPH CLASSES
}

\author{
Laurent Bulteau, ${ }^{1}$ Vincent Froese, ${ }^{2}$ and Nimrod Talmon ${ }^{3}$ \\ ${ }^{1}$ IGM-LabInfo, CNRS UMR 8049, Université Paris-Est Marne-la-Vallée, Paris, \\ France \\ ${ }^{2}$ Institut für Softwaretechnik und Theoretische Informatik, TU Berlin, Berlin, \\ Germany \\ ${ }^{3}$ Weizmann Institute of Science, Rehovot, Israel
}

\begin{abstract}
We study competitive diffusion games on graphs introduced in the literature to model the spread of influence in social networks. Extending results of a prior study for two players, we investigate the existence of pure Nash equilibria for at least three players on different classes of graphs including paths, cycles, grid graphs and hypercubes; as a main contribution, we answer an open question proving that there is no Nash equilibrium for three players on $m \times n$ grids with $\min \{m, n\} \geq 5$. Further extending results of previous studies for two players, we prove the existence of pure Nash equilibria for four players on every d-dimensional hypercube.
\end{abstract}

\section{INTRODUCTION}

Social networks, and the diffusion of information within them, yield an interesting and well-researched field of study. Among other models, competitive diffusion games have been introduced [1] as a game-theoretic approach toward modeling the process of diffusion (or propagation) of influence (or information in general) in social networks. Such models have applications in "viral marketing," where several companies (or brands) compete to influence as many customers (of products) or users (of technologies) as possible by initially selecting only a "small" subset of target users that will "infect" a large number of other users. Herein, the network is modeled as an undirected graph in which the vertices correspond to the users, with edges modeling influence relations between them. The companies, being the players of the corresponding diffusion game, choose an initial subset of target vertices, which then influence other neighboring vertices via a certain propagation process. More concretely, a vertex adopts a company's product at some specific time during the process if he is influenced by (that is, connected by an edge to) another vertex that already adopted this product. After adopting a product of one company, a vertex will never adopt any other product in the future. However, if a vertex gets influenced by several companies at the same time, then it will not adopt any of them and it is removed from the game. See Section 1.3 for the formal definitions of the game.

In their initial work, [1] studied how the existence of pure Nash equilibria is influenced by the diameter of the underlying graph. Following this line of research, [8] investigated the existence of Nash equilibria for competitive diffusion games with two players on

Address correspondence to Vincent Froese, Institut für Softwaretechnik und Theoretische Informatik, TU Berlin, Berlin 10623, Germany. Email: vincent.froese@tu-berlin.de 
several classes of graphs such as paths, cycles, and grid graphs. Notably, she proved that on sufficiently large grids, there always exists a Nash equilibrium for two players, further conjecturing that there is no Nash equilibrium for three players on grids. We extend the results of [8] for two players to three or more players on paths, cycles, and grid graphs, proving the conjectured nonexistence of a pure Nash equilibrium for three players on grids as a main result. Also following this line of research, [3] investigated the existence of Nash equilibria for competitive diffusion games with two players on $d$-dimensional hypercubes. We extend their results by showing that there always exists a Nash equilibrium for four players on any $d$-dimensional hypercube.

An overview of our results is given in Section 1.2. After introducing the preliminaries in Section 1.3, we discuss our results for paths and cycles in Section 2, followed by the proof of our main contribution with respect to grids in Section 3. We discuss hypercubes in Section 4 and finish with some statements concerning general graphs in Section 5.

\subsection{Related Work}

The study of influence maximization in social networks was initiated by [6]. Several game-theoretic models have been suggested, including our model of reference, introduced by [1]. Some interesting generalizations of this model are the model by [12], who considered a more complex underlying diffusion process, and the model studied by [3], allowing each player to choose multiple vertices. The so-called Voronoi games, which are closely related to our model (but not similar; there, players can share vertices), were studied by $[2,7]$. Recently, [4] considered the competitive diffusion game on weighted graphs, including negative weights. Concerning our model, [1] claimed the existence of pure Nash equilibria for any number of players on graphs of diameter at most two; however, [11] gave a counterexample consisting of a graph with nine vertices and diameter two with no Nash equilibrium for two players.

Our main point of reference is the work of [8], who studied the existence (and nonexistence) of pure Nash equilibria, mainly for two players on special graph classes (paths, cycles, trees, unicycles, and grids); indeed, our work can be seen as extending of that work to more than two players. It was already showed [9] that there is a Nash equilibrium for any number of players on any star or clique. There is always a pure Nash equilibrium for two players on a tree as proved by [10], but not always for more than two players. Safe strategies can be considered for trees and spider graphs, where a safe strategy is a strategy that maximizes the minimum payoff of a certain player when the minimum is taken over the possible unknown actions of the other players was.

\subsection{Our Results}

We begin by characterizing the existence of Nash equilibria for paths and cycles, showing that, except for three players on paths of length at least six, a Nash equilibrium exists for any number of players playing on any such graph (Theorems 2.1. and 2.5). We then prove Conjecture 1 of [8], showing that there is no Nash equilibrium for three players on $G_{m \times n}$, as long as both $m$ and $n$ are at least 5 (Theorem 3.1). Then, we prove the existence of Nash equilibria for four players on any $d$-dimensional hypercube (Theorem 4.1). Finally, we investigate the minimum number of vertices such that there is an arbitrary graph with no Nash equilibrium for $k$ players. We prove an upper bound showing that there always exists a tree on $\left\lfloor\frac{3}{2} k\right\rfloor+2$ vertices with no Nash equilibrium for $k$ players (Theorem 5.2). 


\subsection{Preliminaries}

Notation. For $i, j \in \mathbb{N}$ with $i<j$, we define $[i, j]:=\{i, \ldots, j\}$ and $[i]:=$ $\{1, \ldots, i\}$. We consider simple, finite, undirected graphs $G=(V, E)$ with vertex set $V$ and edge set $E \subseteq\{\{u, v\} \mid u, v \in V\}$. For two vertices $u, v \in V$, we define the distance $\operatorname{dist}_{G}(u, v)$ between $u$ and $v$ to be the length of a shortest path from $u$ to $v$ in $G$.

A path $P_{n}=(V, E)$ on $n$ vertices is the graph with $V=[n]$ and $E=\{\{i, i+1\} \mid i \in$ $[n-1]\}$. A cycle $C_{n}=(V, E)$ on $n$ vertices is the graph with $V=[n]$ and $E=\{\{i, i+1\} \mid$ $i \in[n-1]\} \cup\{\{n, 1\}\}$. For $m, n \in \mathbb{N}$, the $m \times n$ grid $G_{m \times n}=(V, E)$ is a graph with vertices $V=[m] \times[n]$ and edges $E=\left\{\left\{(x, y),\left(x^{\prime}, y^{\prime}\right)\right\}|| x-x^{\prime}|+| y-y^{\prime} \mid=1\right\}$. We use the term position for a vertex $v \in V$. Note that the distance of two positions $v=(x, y)$, $v^{\prime}=\left(x^{\prime}, y^{\prime}\right) \in V$ is $\operatorname{dist}_{G_{m \times n}}\left(v, v^{\prime}\right)=\left\|v-v^{\prime}\right\|_{1}:=\left|x-x^{\prime}\right|+\left|y-y^{\prime}\right|$. For $d \in \mathbb{N}, d \geq 1$, the $d$-dimensional hypercube $H_{d}=(V, E)$ is defined on the vertex set $V=\{0,1\}^{d}$, that is, a vertex $x=x_{1} \ldots x_{d} \in V$ is a binary string of length $d$. The set of edges is defined as $E=\{\{x, y\} \mid \Delta(x, y)=1\}$, where $\Delta(x, y):=\left|\left\{i \in[d] \mid x_{i} \neq y_{i}\right\}\right|$ is the Hamming distance of $x$ and $y$, that is, the number of positions in which $x$ and $y$ have different bits. Note that $\operatorname{dist}_{H_{d}}(x, y)=\Delta(x, y)$.

Diffusion game on graphs. A competitive diffusion game $\Gamma=(G, k)$ is defined by an undirected graph $G=(V, E)$ and a number $k$ of players (we name the players Player $1, \ldots$, Player $k$ ), each having its distinct color in $[k]$. The strategy space of each player is $V$, such that each player $i$ selects a single vertex $v_{i} \in V$ at time 0 , which is then colored by the color $i$. If two players choose the same vertex $v$, then this vertex is removed from the graph. For Player $i$, we use the terms strategy and position interchangeably to mean its chosen vertex. A strategy profile is a tuple $\left(v_{1}, \ldots, v_{k}\right) \in V^{k}$ containing the initially chosen vertex for each player. The payoff $U_{i}\left(v_{1}, \ldots, v_{k}\right)$ of Player $i$ is the number of vertices with color $i$ after the following propagation process. At time $t+1$, any so-far uncolored vertex that has only uncolored neighbors and neighbors colored in $i$ (and no neighbors with other colors $j \in[k] \backslash\{i\}$ ) is colored in $i$. Any uncolored vertex with at least two different colors among its neighbors is removed from the graph. The process terminates when the coloring of the vertices does not change between consecutive steps. A strategy profile $\left(v_{1}, \ldots, v_{k}\right)$ is a (pure) Nash equilibrium if, for any player $i \in[k]$ and any vertex $v^{\prime} \in V$, it holds that $U_{i}\left(v_{1}, \ldots, v_{i-1}, v^{\prime}, v_{i+1}, \ldots, v_{k}\right) \leq U_{i}\left(v_{1}, \ldots, v_{k}\right)$.

\section{PATHS AND CYCLES}

In this section, we fully characterize the existence of Nash equilibria on paths and cycles, for any number $k$ of players.

Theorem 2.1. For any $k \in \mathbb{N}$ and any $n \in \mathbb{N}$, there is a Nash equilibrium for $k$ players on $P_{n}$, except for $k=3$ and $n \geq 6$.

The general idea of the proof is to pair the players and distribute these pairs evenly. In the rest of this section, we prove three Lemmas whose straightforward combination proves Theorem 2.1.

Lemma 2.2. For any even $k \in \mathbb{N}$ and any $n \in \mathbb{N}$, there is a Nash equilibrium for $k$ players on $P_{n}$. 


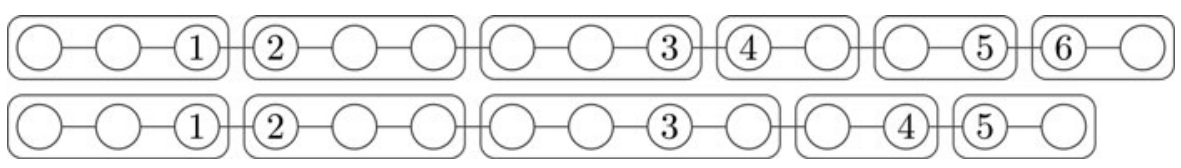

Figure 1 Illustrations for Theorem 2.1, showing a Nash equilibrium for six players on $P_{15}$ (top) and a Nash equilibrium for five players on $P_{14}$ (bottom). The boxes show the colored regions of each player.

Proof. If $n \leq k$, then a strategy profile in which each vertex of the path is chosen by at least one player is clearly a Nash equilibrium.

Otherwise, if $n>k$, then the idea is to build pairs of players, which are then placed such that two paired players are neighbors, and the distance of any two consecutive pairs is roughly equal (specifically, differs by at most two). See Figure 1 for an example. Intuitively, this yields a Nash equilibrium because each player obtains roughly the same payoff (specifically, differing by at most one), therefore, no player can improve. Because we have $n$ vertices, we want each player's payoff to be at least $z:=\left\lfloor\frac{n}{k}\right\rfloor$. This leaves $r:=n$ mod $k$ other vertices, which we distribute between the first $r$ players such that the payoff of any player is at most $z+1$. This can be achieved as follows. Let $p_{i} \in[n]$ denote the position of Player $i$, that is, the index of the chosen vertex on the path. We define

$$
p_{i}:= \begin{cases}z \cdot i+\min \{i, r\} & \text { if } i \text { is odd } \\ p_{i-1}+1 & \text { if } i \text { is even. }\end{cases}
$$

Note that, by construction, it holds that $p_{1} \in\{z, z+1\}$ and $p_{k}=n-z+1$. Moreover, for each odd index $i \geq 3$, we have

$$
\begin{aligned}
p_{i}-p_{i-1} & =z i+\min \{i, r\}-(z(i-2)+\min \{i-2, r\}+1) \\
& =2 z+\min \{i, r\}-\min \{i-2, r\}-1 .
\end{aligned}
$$

Note that $\min \{i, r\}-\min \{i-2, r\} \in\{0,1,2\}$ and thus $2 z-1 \leq p_{i}-p_{i-1} \leq 2 z+1$. We claim that $u_{i}:=U_{i}\left(p_{1}, \ldots, p_{k}\right) \in\{z, z+1\}$ holds for each $i \in[k]$. Clearly, $u_{1}=$ $p_{1} \in\{z, z+1\}$ and $u_{k}=n-p_{k}+1=z$. For all odd $i \geq 3$, it is not difficult to see that $u_{i}=u_{i-1}=1+\left\lfloor\left(p_{i}-p_{i-1}-1\right) / 2\right\rfloor \in\{z, z+1\}$, which proves the claim.

To see that the strategy profile $\left(p_{1}, \ldots, p_{k}\right)$ is a Nash equilibrium, consider an arbitrary Player $i$ and any other strategy $p_{i}^{\prime} \in[n]$ that is picked. Clearly, we can assume $p_{i}^{\prime} \neq p_{j}$ for all $j \neq i$ since, otherwise, Player $i$ 's payoff is zero. If $p_{i}^{\prime}<p_{1}$ or $p_{i}^{\prime}>p_{k}$, then Player $i$ gets a payoff of at most $z$. If $p_{j}<p_{i}^{\prime}<p_{j+1}$ for some even $j \in[2, k-2]$, then the payoff is at most $1+\left\lfloor\left(p_{j+1}-p_{j}-2\right) / 2\right\rfloor \leq z$.

We can modify the construction given in the proof of Lemma 2.2 to also work for odd numbers $k$ greater than three.

Lemma 2.3. For any odd $k>3 \in \mathbb{N}$ and for any $n \in \mathbb{N}$, there is a Nash equilibrium for $k$ players on $P_{n}$.

Proof. We give a strategy profile based on the construction for an even number of players (proof of Lemma 2.2). The idea is to pair the players, placing the remaining lonely player between two consecutive pairs. 
This is best explained using a reduction to the even case. Specifically, given the strategy profile $\left(p_{1}^{\prime}, \ldots, p_{k+1}^{\prime}\right)$ for an even number $k+1$ of players on $P_{n+1}$ as constructed in the proof of Lemma 2.2 , we define the strategy profile $\left(p_{1}, \ldots, p_{k}\right):=\left(p_{1}^{\prime}, \ldots, p_{k-2}^{\prime}, p_{k}^{\prime}-\right.$ $\left.1, p_{k+1}^{\prime}-1\right)$. To see why this results in a Nash equilibrium, let $z:=\lfloor(n+1) /(k+1)\rfloor$ and note that, by construction, it holds that $p_{1} \in\{z, z+1\}, p_{k}=n-z+1$, and $2 z-1 \leq$ $p_{i+1}-p_{i} \leq 2 z+1$ for all $i \in[2, k-1]$. Moreover, each player receives a payoff of at least $z$, therefore, all players (except for Player $(k-2)$ ) cannot improve by the same arguments as those in the proof of Lemma 2.2. Regarding Player $(k-2)$, note that the payoff is

$$
1+\left\lfloor\left(p_{k-1}-p_{k-2}-1\right) / 2\right\rfloor+\left\lfloor\left(p_{k-2}-p_{k-3}-1\right) / 2\right\rfloor \geq 2 z-1 .
$$

Hence, it clearly cannot be improved by choosing any position outside of $\left[p_{k-3}, p_{k-1}\right]$. Also, it cannot be improved by choosing any other position in $\left[p_{k-3}, p_{k-1}\right]$. To see this, note that the maximum payoff from any position in $\left[p_{k-3}, p_{k-1}\right]$ is

$$
1+\left\lfloor\left(p_{k-1}-p_{k-3}-2\right) / 2\right\rfloor=1+\left\lfloor\left(p_{k-1}-p_{k-2}-1+p_{k-2}-p_{k-3}-1\right) / 2\right\rfloor,
$$

which is equal to the aforementioned payoff because $p_{k-1}-p_{k-2}$ and $p_{k-2}-p_{k-3}$ cannot both be even, by construction.

It remains to discuss the fairly simple (non)existence of Nash equilibria for three players. Note that [8] already stated without proof that there is no Nash equilibrium for three players on $G_{2 \times n}$ and $G_{3 \times n}$ and [10] showed that there is no Nash equilibrium for three players on $P_{7}$. For the sake of completeness, we prove the following lemma.

Lemma 2.4. For three players, there is a Nash equilibrium on $P_{n}$ if and only if $n \leq 5$.

Proof. If $n \leq 3$, then a strategy profile in which each vertex of the path is chosen by at least one player is clearly a Nash equilibrium. For $n \in\{4,5\}$, the strategy profile $(2,3,4)$ is a Nash equilibrium.

To see that there is no Nash equilibrium for $n \geq 6$, consider an arbitrary strategy profile $\left(p_{1}, p_{2}, p_{3}\right)$. Without loss of generality, we can assume that $p_{1}<p_{2}<p_{3}$ and consider the following two cases. First, we assume that $p_{2}=p_{1}+1$ and $p_{3}=p_{2}+1$. If $p_{1}>2$, then Player 2 increases the payoff by choosing $p_{1}-1$. Otherwise, it holds that $p_{3}<n-1$, and Player 2 increases the payoff by moving to $p_{3}+1$. Therefore, this case does not yield a Nash equilibrium. For the remaining case, it holds that $p_{1}<p_{2}-1$ or $p_{3}>p_{2}+1$. If $p_{1}<p_{2}-1$, then Player 1 increases his/her payoff by moving to $p_{2}-1$, whereas if $p_{3}>p_{2}+1$, then Player 3 increases his/her payoff by moving to $p_{2}+1$. Thus, this case does not yield a Nash equilibrium as well, and we are done.

We close this section with the following result considering cycles. Interestingly, for cycles there exists a Nash equilibrium also for three players.

Theorem 2.5. For any $k, n \in \mathbb{N}$, there is a Nash equilibrium for $k$ players on $C_{n}$.

Proof. It is an easy observation that the constructions given in the proofs of Lemmas 2.2 and 2.3 also yield Nash equilibria for cycles, that is, when the two endpoints of the path are connected by an edge. Thus, it remains to show a Nash equilibrium for $k=3$ players for 
any $C_{n}$. We set $p_{1}:=1, p_{2}:=n$ and

$$
p_{3}:= \begin{cases}\lfloor n / 2\rfloor & \text { if } n \bmod 4=1, \\ \lceil n / 2\rceil & \text { else. }\end{cases}
$$

It is not difficult to check that $\left(p_{1}, p_{2}, p_{3}\right)$ is a Nash equilibrium.

\section{GRID GRAPHS}

In this section we consider three players on the $m \times n$ grid $G_{m \times n}$ and we prove the following theorem.

Theorem 3.1. If $n \geq 5$ and $m \geq 5$, then there is no Nash equilibrium for three players on $G_{m \times n}$.

Before proving the theorem, let us first introduce some general definitions and observations. Throughout this section, we denote the strategy of Player $i$, that is, the initially chosen vertex of Player $i$, by $p_{i}:=\left(x_{i}, y_{i}\right) \in[m] \times[n]$. Note that any strategy profile in which more than one player chooses the same position is never a Nash equilibrium, because in this case, each of these players gets a payoff of zero and can improve his/her payoff by choosing any free vertex (to obtain a payoff of at least one). Therefore, we will assume w.l.o.g. that $p_{1} \neq p_{2} \neq p_{3}$. Further, note that the game is highly symmetric with respect to the axes. Specifically, reflecting coordinates along a dimension or rotating the grid by 90 degrees yields the same outcome for the game. Thus, in what follows, we consider only possible cases up to the above symmetries.

We define $\Delta_{x}:=\max _{i, j \in[k]}\left|x_{i}-x_{j}\right|$ and $\Delta_{y}:=\max _{i, j \in[k]}\left|y_{i}-y_{j}\right|$ to be the maximum coordinate-wise differences among the positions of the players. We say that a player strictly controls the other two players if both reside on the same side of the player, in both dimensions.

Definition 3.2. Player $i$ strictly controls the other players, if for each other Player $j$ with $j \neq i$, either

$$
\begin{aligned}
& \left(x_{i}<x_{j}\right) \wedge\left(y_{i}<y_{j}\right), \text { or } \\
& \left(x_{i}<x_{j}\right) \wedge\left(y_{i}>y_{j}\right) \text { or } \\
& \left(x_{i}>x_{j}\right) \wedge\left(y_{i}<y_{j}\right), \text { or } \\
& \left(x_{i}>x_{j}\right) \wedge\left(y_{i}>y_{j}\right) \text { holds. }
\end{aligned}
$$

We now describe the outline for the proof of Theorem 3.1.

Proof of Theorem 3.1 Let $m \geq 5$ and $n \geq 5$. We perform a case distinction based on the relative positions of the three players. As a first case, we consider strategy profiles wherein the players are playing "far" from each other, that is, there are two players whose positions differ by at least three in some coordinate (formally $\max \left\{\Delta_{x}, \Delta_{y}\right\} \geq 3$ ). For these profiles, we distinguish two subcases: there exist a player who strictly controls the others (Lemma 3.5) or there does not exist a player who strictly controls the others (Lemma 3.6). 
We prove that none of these cases yields a Nash equilibrium by showing that there always exists a player who can improve the payoff. Notably, the improving player always moves closer to the other two players. We are left with the case where the players are playing "close" to each other, in the sense that their positions all lie inside a $3 \times 3$ subgrid (that is, $\max \left\{\Delta_{x}, \Delta_{y}\right\} \leq 2$ ). For these strategy profiles, we show that there always exists a player who can improve the payoff (Lemma 3.7), however, the improving position depends not only on the relative positions between the players, but also on the global positioning of this subgrid on the overall grid. This leads to a somewhat erratic behavior, which we overcome by considering all possible close positions (up to symmetries) in the proof of Lemma 3.7. Altogether, Lemmas 3.5, 3.6 and 3.7 cover all possible strategy profiles (ruling them out as Nash equilibria), thus implying the theorem.

In order to conclude Theorem 3.1, it remains to prove the lemmas mentioned in the case distinction discussed previously. To this end, we start with two easy preliminary results. First, we observe that for a vertex for which there is a unique player, the shortest distance to it is colored in that player's color (note that this is true in general for every graph and any number of players).

Observation 3.3. Let $G=(V, E)$ be an undirected graph and let $\left(p_{1}, \ldots, p_{k}\right)$ be a strategy profile. Let $v \in V$ be a vertex for which there exists an $i \in[k]$ such that $\operatorname{dist}_{G}\left(p_{i}, v\right)=: \delta<\operatorname{dist}\left(p_{j}, v\right)$ holds for all $j \in[k], j \neq i$, then $v$ will be colored in color $i$ at time $\delta$.

Proof. The proof is by induction on the distance $\delta$. For $\delta=0$, it clearly follows from the definition of the propagation process that $v=p_{i}$ has color $i$ at time 0 . For all $\delta>0$, it follows from the induction hypothesis that $v$ has a neighbor $u$ with $\operatorname{dist}\left(p_{i}, u\right)=\delta-1$ that is colored in color $i$ at time $\delta-1$. Moreover, for all neighbors $w$ of $v$, it holds $\operatorname{dist}\left(p_{j}, w\right)>\delta-1$ for all $j \neq i$. This implies that no neighbor of $v$ has a different color than $i$ at time $\delta-1$, and thus, $v$ has color $i$ at time $\delta$.

Based on Observation 3.3, we show that whenever a player has distance at least three to the other players and both of them are positioned on the same side of that player (with respect to both dimensions), then the payoff can be improved by moving closer to the others (see Figure 2 for an illustration).

Proposition 3.4. If $x_{1} \leq x_{j}, y_{1} \leq y_{j}$, and $\left\|p_{1}-p_{j}\right\|_{1} \geq 3$ holds for $j \in\{2,3\}$, then Player 1 can increase his/her payoff by moving to $\left(x_{1}+1, y_{1}+1\right)$.

Proof. Let $p_{1}^{\prime}:=\left(x_{1}+1, y_{1}+1\right)$ and $x \in\left[x_{1}\right] \times\left[y_{1}\right]$. Note that $\left\|p_{1}^{\prime}-x\right\|_{1}=\left\|p_{1}-x\right\|_{1}+2<$ $\left\|p_{j}-x\right\|_{1}=\left\|p_{1}-p_{j}\right\|_{1}+\left\|p_{1}-x\right\|_{1} \geq\left\|p_{1}-x\right\|_{1}+3$ holds for $j \in\{2,3\}$. Hence, Player 1 still has the unique shortest distance to $x$. By Observation 3.3, $x$ is colored with color 1 . Moreover, for any other position $x \notin\left[x_{1}\right] \times\left[y_{1}\right]$, there is a shortest path from $p_{1}$ to $x$ going through at least one of the positions $\left(x_{1}+1, y_{1}\right),\left(x_{1}, y_{1}+1\right)$, or $p_{1}^{\prime}$. Clearly, there is also a shortest path from $p_{1}^{\prime}$ to $x$ of at most the same length going through one of these positions. Thus, if $x$ was colored with color 1 before, then $x$ is still colored in color 1 .

To see that Player 1 strictly increases the payoff, note that $\left\|p_{1}^{\prime}-x\right\|_{1}=\left\|p_{1}-x\right\|_{1}-2$ holds for all $x \in\left[x_{1}+1, n\right] \times\left[y_{1}+1, m\right]$. Hence, Player 1 now has the unique shortest distance to all those positions where the distance from $p_{1}$ was at most one larger than the 


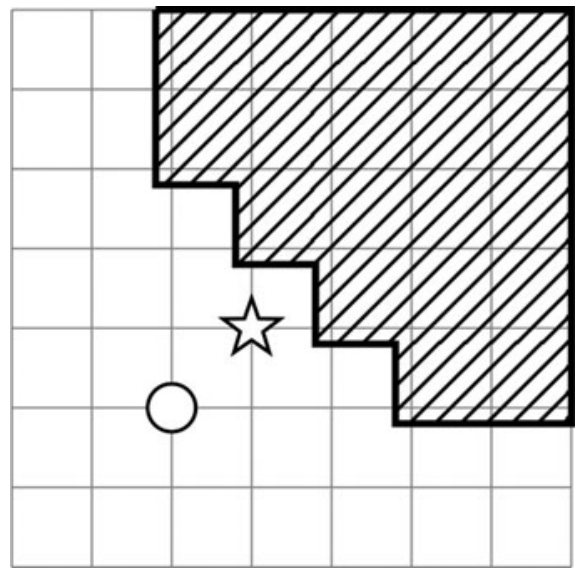

Figure 2 Example of a strategy profile in which Player 1 (white circle) has both other players to his/her top right with distance at least three (the shaded region denotes the possible positions for Player 2 and 3). Player 1 can increase his/her payoff by moving closer to the others (star).

shortest distance from any other player (clearly, there exists at least one such position with color $j \neq 1$ ). By Observation 3.3, these positions now get color 1, thus, Player 1 strictly increases the payoff.

We go on to prove the lemmas, starting with the case that the players play far from each other. The following lemma handles the first subcase, that is, when one of the players strictly controls the others.

Lemma 3.5. A strategy profile with $\max \left\{\Delta_{x}, \Delta_{y}\right\} \geq 3$ in which one of the players strictly controls the others is not a Nash equilibrium.

Proof. We assume w.l.o.g. that Player 1 strictly controls Player 2 and Player 3; specifically, we assume that $x_{1}<x_{2}$ and $y_{1}<y_{2}$ and $x_{1}<x_{3}$ and $y_{1}<y_{3}$ holds. Figure 3 depicts the

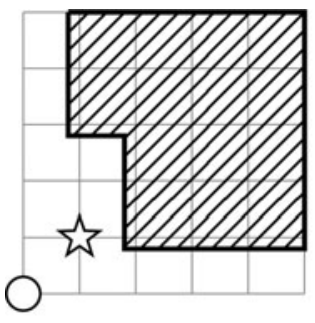

1

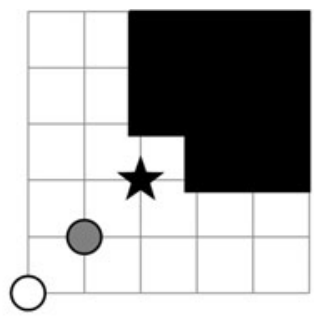

$2(\mathrm{a})$

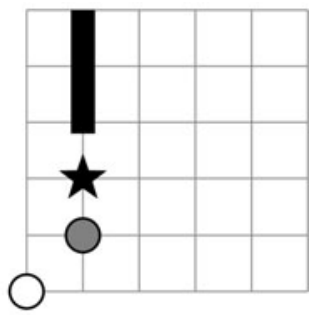

2(b)

Figure 3 Possible cases (up to symmetry) for Player 1 (white) strictly controlling Player 2 (gray) and Player 3 (black). Circles denote the players' strategies. The shaded region contains the possible positions of both Players 2 and 3, whereas the black regions denote possible positions for Player 3 only. A star marks the position improving the payoff of the respective player. 
three possible cases for the positions of Player 2 and Player 3. For each case, we show that a player who can improve the payoff exists.

Case 1: We assume that $\left(x_{2}, y_{2}\right) \neq\left(x_{1}+1, y_{1}+1\right)$ and $\left(x_{3}, y_{3}\right) \neq\left(x_{1}+1, y_{1}+1\right)$. By Proposition 3.4, Player 1 gets a higher payoff from $\left(x_{1}+1, y_{1}+1\right)$.

Case 2: We assume w.l.o.g. that $\left(x_{2}, y_{2}\right)=\left(x_{1}+1, y_{1}+1\right)$.

(a) We assume $x_{2}<x_{3}$ and $y_{2}<y_{3}$. Then, $x_{3}>x_{2}+1$ or $y_{3}>y_{2}+1$ holds, since $\max \left\{\Delta_{x}, \Delta_{y}\right\} \geq 3$. Note that Player 3 strictly controls Player 1 and Player 2 and that this case is symmetric to Case 1 .

(b) We assume $x_{2} \geq x_{3}$ or $y_{2} \geq y_{3}$. Then, it holds that $x_{3}=x_{2}$ or $y_{3}=y_{2}$. We assume $x_{3}=x_{2}$ (the argument for $y_{3}=y_{2}$ being analogous). Since $\max \left\{\Delta_{x}, \Delta_{y}\right\} \geq 3$, we have $y_{3}>y_{2}+1$, thus Player 3 can improve by moving to $\left(x_{2}, y_{2}+1\right)$ because then all positions in $[m] \times\left[y_{2}+1, n\right]$ are colored in color 3 , and before, only a strict subset of these positions was colored in color 3 .

The other subcase, in which no player strictly controls the others, is handled by the following lemma.

Lemma 3.6. A strategy profile with $\max \left\{\Delta_{x}, \Delta_{y}\right\} \geq 3$ in which no player strictly controls the others is not a Nash equilibrium.

Proof. If no player strictly controls the others, then it follows that at least two players have the same coordinate in at least one dimension. We perform a case distinction on the cases as depicted in Figure 4.

Case 1: All three players have the same coordinate in one dimension. We assume that $x_{1}=$ $x_{2}=x_{3}$ (the case $y_{1}=y_{2}=y_{3}$ is analogous). Without loss of generality also $y_{1}<y_{2}<y_{3}$ holds. Since $\max \left\{\Delta_{x}, \Delta_{y}\right\} \geq 3$, it follows that $y_{i+1}-y_{i} \geq 2$ holds for some $i \in\{1,2\}$, say for $i=2$. Clearly, Player 3 can improve his/her payoff by choosing $\left(x_{3}, y_{2}+1\right)$ (analogous to Case $2 \mathrm{~b}$ in the proof of Lemma 3.5).

Case 2: There is a dimension where two players have the same coordinate but not all three players have the same coordinate in any dimension. We assume $x_{1}=x_{2}<x_{3}$ and $y_{1}<y_{2}$ (all other cases are analogous). We also assume that $y_{1} \leq y_{3} \leq y_{2}$, since, otherwise, Player 3 strictly controls the others, and this case is handled by Lemma 3.5.

(a) We assume that $y_{2}=y_{1}+1$. Then, $x_{3} \geq x_{1}+3$ holds since $\max \left\{\Delta_{x}, \Delta_{y}\right\} \geq 3$. Player 3 increases his/her payoff by moving to $\left(x_{1}+2, y_{1}\right)$ (analogous to Case $2 b$ in the proof of Lemma 3.5).

(b) We assume that $y_{2}=y_{1}+2$. Then, $x_{3} \geq x_{1}+3$ holds since $\max \left\{\Delta_{x}, \Delta_{y}\right\} \geq 3$. Player 3 increases his/her payoff by moving to $\left(x_{1}+2, y_{1}+1\right)$ (analogous to Case $2 b$ in the proof of Lemma 3.5).

(c) We assume that $y_{2}>y_{1}+2$ and $\left|y_{2}-y_{3}\right| \leq\left|y_{1}-y_{3}\right|$. That is, w.l.o.g. Player 3 is closer to Player 2. Then, by Proposition 3.4, Player 1 increases his/her payoff by moving to $\left(x_{1}+1, y_{1}+1\right)$.

It remains to consider the cases when the players play close to each other. 


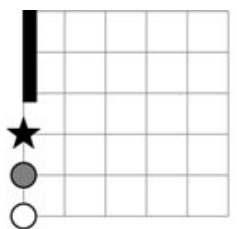

1

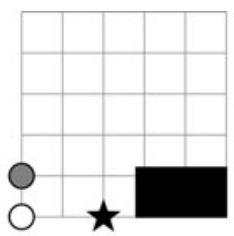

2(a)

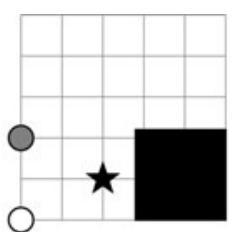

2(b)

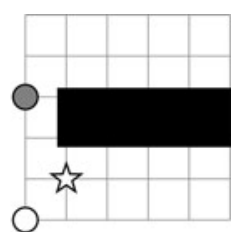

$2(\mathrm{c})$

Figure 4 Possible cases (up to symmetry) when no player strictly controls the others. Circles denote the positions of Player 1 (white) and Player 2 (gray). The black regions contain the possible positions for Player 3. A star marks the position improving the payoff of the respective player.

Lemma 3.7. A strategy profile with $\max \left\{\Delta_{x}, \Delta_{y}\right\} \leq 2$ is not a Nash equilibrium.

Proof. First, we assume that $\Delta_{x}+\Delta_{y} \geq 2$, because otherwise, there would be at least two players on the same position (so each of them can improve by moving to any free vertex). Without loss of generality, we also assume that $\Delta_{x} \leq \Delta_{y}$, leaving the following cases to consider (depicted in Figure 5).

Case 1: Let $\left(x_{1}, y_{1}\right)=(x, y) \in[m] \times[n-2],\left(x_{2}, y_{2}\right)=(x, y+1)$, and $\left(x_{3}, y_{3}\right)=(x, y+2)$ be the positions of the three players. Clearly, with these positions, Player 1 gets a payoff of $m y$, Player 2's payoff is $m$, and Player 3 gets a payoff of $m(n-y-1)$. Now, if $y \geq 3$, then Player 2 can improve by choosing ( $x, y-1)$, thus achieving a payoff of $m(y-1)>m$. If $y=1$, then Player 2 improves by playing on $(x, y+3)$ with a payoff of $m(n-y-2)>m$ (remember that $n \geq 5$ ). Also, if $y=2$ and $n>5$, then Player 2 gets a higher payoff by choosing $(x, y+3)$. For $y=2$ and $n=5$, we observe that either $x \geq\lceil m / 2\rceil$ or $m-x \geq\lceil m / 2\rceil$ and assume, w.l.o.g. that $x \geq\lceil m / 2\rceil$ holds. Then, applying Observation 3.3, it holds that, if Player 2 chooses $(x-1, y)$, then all positions in $[x-1] \times[y+1]$ are colored
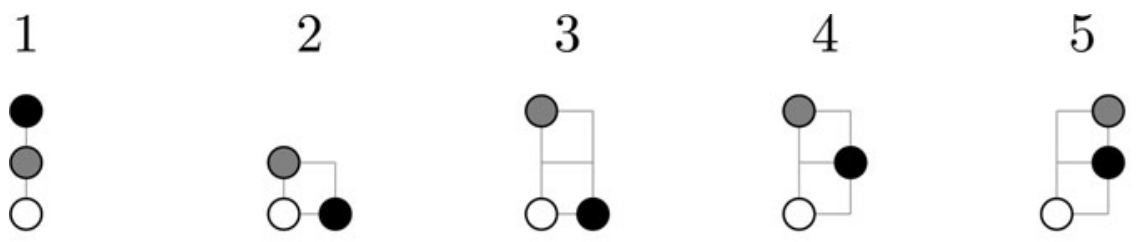

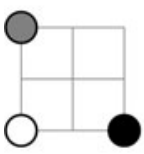

6

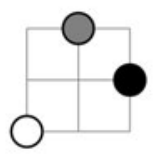

7

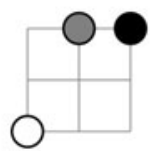

8

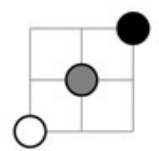

9

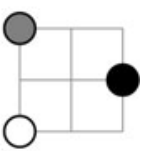

10

Figure 5 Possible positions (up to symmetry) of three players playing inside a subgrid of size at most $3 \times 3$. The position of Player 1 (white) is denoted $(x, y)$. 
in color 2, Thus, the payoff of Player 2 is at least $(\lceil m / 2\rceil-1) 3>m$ (remember that $m \geq 5$ ).

Case 2: Let $\left(x_{1}, y_{1}\right)=(x, y) \in[m-1] \times[n-1],\left(x_{2}, y_{2}\right)=(x, y+1)$, and $\left(x_{3}, y_{3}\right)=$ $(x+1, y)$ be the positions of the three players. Note that, due to symmetry, this is the only case we have to consider (w.l.o.g.). Clearly, exactly the positions in $[x] \times[y]$ are colored in color 1, therefore Player 1 has a payoff of $x y$. If $x<m / 2$, then position $(x+2, y)$ yields a payoff of $(m-x-1) n \geq(\lceil m / 2\rceil-1) n$ for Player 1 since Player 1 colors all vertices in $[x+2, m] \times[n]$. Note that $\lceil m / 2\rceil-1 \geq x$ and $n>y$, thus, Player 1's payoff improved. Analogously, for $y<n / 2$, Player 1 's payoff from position $(x, y+2)$ is at least $m(\lceil n / 2\rceil-1)>x y$. If $x>\lceil m / 2\rceil$, then Player 3 can improve by choosing $(x-1, y)$. To see that this is true, note first that by choosing $\left(x_{3}, y_{3}\right)$, Player 3 colors only positions in $[x+1, m] \times[n]$. Now, observe that if position $\left(x^{\prime}, y^{\prime}\right)$ is colored in color 3 when Player 3 chooses $\left(x_{3}, y_{3}\right)$, then it holds also that position $\left(x-\left(x^{\prime}-x\right), y\right)$ is colored in color 3 when Player 3 chooses $(x-1, y)=\left(x-\left(x_{3}-x\right), y\right)$, due the symmetries, and because the distances from this position to the players, positions are all identical. Hence, Player 3 colors at least the same number of positions in $[x-(m-x), x-1] \times[y]$. Note that $x-(m-x) \geq 2$ since $x>\lceil m / 2\rceil$. By Observation 3.3, Player 3 additionally colors position $(1,1)$, yielding a strictly greater payoff. By analogous arguments, for $y>\lceil n / 2\rceil$, the position $(x, y-1)$ yields a better payoff for Player 2. Finally, assume $x=\lceil m / 2\rceil$ and $y=\lceil n / 2\rceil$. Now, Player 1 can improve by choosing $(x-1, y+1)$, thus coloring at least all positions in $[x-1] \times[n]$, giving a payoff of at least $(\lceil m / 2\rceil-1) n>\lceil m / 2\rceil \cdot\lceil n / 2\rceil=x y$ for $m \geq 5$ and $n \geq 5$.

Case 3: Let $\left(x_{1}, y_{1}\right)=(x, y) \in[m-1] \times[n-2],\left(x_{2}, y_{2}\right)=(x, y+2)$, and $\left(x_{3}, y_{3}\right)=$ $(x+1, y)$ be the positions of the three players. First, note that Player 1 colors all positions in $[x] \times[y]$, gaining a payoff of $x y$, Player 2 colors $[m] \times[y+2, n]$, gaining a payoff of $m(n-y-1)$, and Player 3 gets all positions in $[x+1, n] \times$ $[y+1]$, gaining a payoff of exactly $(m-x)(y+1)$. Now, if $y=1$, then Player 1 can choose $(x, 4)$ to obtain a payoff of $m(n-3)>x$, since $m>x$ and $n-3 \geq 2$. Hence, assume $y>1$, and observe that both the payoff of Player 1 from $(x+1, y-1)$ and the payoff of Player 3 from $(x, y-1)$ equals $m(y-1)$. Assuming that we have a Nash equilibrium, we obtain the two inequations $x y \geq m(y-1)$ and $(m-x)(y+1) \geq m(y-1)$, which yield $m(y-1) / y \leq x \leq 2 m /(y+1)$. Note that we obtain a contradiction for $y \geq 3$. Hence, we can assume that $y=2$ and $\lceil m / 2\rceil \leq x \leq\lfloor 2 m / 3\rfloor$. If $n \geq 6$, then Player 1 can improve by choosing $(x, 5)$ achieving a payoff of $m(n-4) \geq 2 m>2 x$. Thus, we also assume $n=5$. Now, Player 1 can choose position $(x-1,4)$ to color all but three positions in $[x-1] \times[5]$. The only positions that she does not color are $(x-1,2),(x-1,1)$, and $(x-2,1)$. The payoff is thus $5(x-1)-3$, which, for all $x \geq\lceil m / 2\rceil \geq 3$, is more than $2 x$.

Case 4: Let $\left(x_{1}, y_{1}\right)=(x, y) \in[m-1] \times[n-2],\left(x_{2}, y_{2}\right)=(x, y+2)$, and $\left(x_{3}, y_{3}\right)=$ $(x+1, y+1)$ be the positions of the three players. It is easy to see that, if $x=m-1$, then Player 3's payoff is exactly one, and more can be gained by choosing $(x-1, y+1)$ instead. For $x<m-1$, note that, apart from $\left(x_{3}, y_{3}\right)$, Player 3 colors only positions $\left(x^{\prime}, y^{\prime}\right)$ with $x^{\prime} \geq x_{3}+1=x+2$. Note also that Player 3 does not color all of these positions. For example, at least one of the positions $(x+2, y-1)$ or $(x+2, y+3)$ exists on the grid (since $n \geq 5$ ) 
and is reached by Player 1 or Player 2 at the same time during the propagation process of the game. However, by choosing $(x+2, y+1)$, Player 3 still colors the position $\left(x_{3}, y_{3}\right)$ and clearly all positions $\left(x^{\prime}, y^{\prime}\right)$ with $x^{\prime} \geq x+2$, thus improving the payoff.

Case 5: Let $\left(x_{1}, y_{1}\right)=(x, y) \in[m-1] \times[n-2],\left(x_{2}, y_{2}\right)=(x+1, y+2)$, and $\left(x_{3}, y_{3}\right)=(x+1, y+1)$ be the positions of the three players. If $y=n-2$, then Player 3's payoff is exactly $m$. Therefore, the payoff is increased by moving to $(y-1, x)$, because then the payoff is at least $2 m$. Thus, we can assume that $y<n-2$. If $x \geq m-2$, then Player 2's payoff is either 1 or 3 (it is 1 if $x=m-1$ and 3 if $x=m-2)$. Therefore, the payoff is increased by moving to $(x-2, y)$, because then the payoff is at least $n$ (and $n \geq 5$ ). Thus, we can assume that $x<m-2$. We are left only with the case when $y<n-2$ and $x<m-2$. In this case, Player 2 increases his/her payoff by moving to $(x+2, y+2)$.

Case 6: Let $\left(x_{1}, y_{1}\right)=(x, y) \in[m-2] \times[n-2],\left(x_{2}, y_{2}\right)=(x, y+2)$, and $\left(x_{3}, y_{3}\right)=$ $(x+2, y)$ be the positions of the three players. It is clear that only a strict subset of the positions in $[x+2, m] \times[n]$ are colored in color 3 (for example, the position $(x+2, y+2)$ is not colored in color 3$)$. By choosing $(x+2, y+1)$, however, all positions in $[x+2, m] \times[n]$ are colored in color 3 , resulting in a strictly higher payoff for Player 3.

Case 7: Let $\left(x_{1}, y_{1}\right)=(x, y) \in[m-2] \times[n-2],\left(x_{2}, y_{2}\right)=(x+1, y+2)$, and $\left(x_{3}, y_{3}\right)=(x+2, y+1)$ be the positions of the three players. By Proposition 3.4, Player 1 increases the payoff by moving to $\left(x_{1}+1, y_{1}+1\right)$.

Case 8: Let $\left(x_{1}, y_{1}\right)=(x, y) \in[m-2] \times[n-2],\left(x_{2}, y_{2}\right)=(x+1, y+2)$, and $\left(x_{3}, y_{3}\right)=(x+2, y+2)$ be the positions of the three players. By Proposition 3.4, Player 1 increases the payoff by moving to $\left(x_{1}+1, y_{1}+1\right)$.

Case 9: Let $\left(x_{1}, y_{1}\right)=(x, y) \in[m-2] \times[n-2],\left(x_{2}, y_{2}\right)=(x+1, y+1)$, and $\left(x_{3}, y_{3}\right)=(x+2, y+2)$ be the positions of the three players. Notice that the payoff of Player 2 is only one. It is clear that Player 2 increases the payoff more by moving to $(x, y+1)$, because then the payoff is at least two, because Player 2 also colors the position $(x, y+2)$.

Case 10: Let $\left(x_{1}, y_{1}\right)=(x, y) \in[m-2] \times[n-2],\left(x_{2}, y_{2}\right)=(x, y+2)$, and $\left(x_{3}, y_{3}\right)=$ $(x+2, y+1)$ be the positions of the three players. Note first that Player 1 colors exactly the positions in $[x+1] \times[y]$, thus, that payoff is $(x+1) y$. For $y=1$, Player 1 can move to $(x, 4)$, achieving a payoff of at least $(x+1)(n-3) \geq 2(x+1)$ because he/she colors all positions in $[x+1] \times[4, n]$. Otherwise, if $y \geq 2$, then, by choosing $(x+1, y)$, Player 1 still colors all positions in $[x+1] \times[y]$, and additionally also the position $(x+2, y-1)$.

\section{HYPERCUBES}

Diffusion games on hypercubes have been studied [3] and the existence of a Nash equilibrium for two players on every $d$-dimensional hypercube have been proved [3]. In this section, we extend that result to four players.

Recall that the vertices of $H_{d}$ are all binary strings of length $d$, where two vertices are adjacent if and only if they differ in exactly one bit. Moreover, the geodesic distance of two vertices $u$ and $v$ is exactly the Hamming distance $\Delta(u, v)$. By $\bar{a}$, we denote the complement of $a=a_{1} \ldots a_{d}$, where $\bar{a}_{i}:=1-a_{i}$ for all $i \in[d]$.

We prove the following theorem. 
Theorem 4.1. Let $x, y \in\{0,1\}^{d}$ be two adjacent vertices of $H_{d}$ for $d \geq 1$. Then, every strategy profile $\left(p_{1}, \ldots, p_{4}\right)$ with $\left\{p_{1}, \ldots, p_{4}\right\}=\{x, \bar{x}, y, \bar{y}\}$ is a Nash equilibrium.

A first step to prove Theorem 4.1 is to show that, for a strategy profile of the form as described in Theorem 4.1, it holds that whenever a single player chooses another position, then, for the resulting strategy profile, the payoff of each player equals exactly the number of vertices that has a unique closest distance. Note that we already know from Observation 3.3 that each player always colors all the vertices to which he/she is uniquely closest. In Lemma 4.2, we will show the opposite direction: no player obtains vertices that are not of the unique closest distance among all players (note that this does not hold in general). In a second step, we can then compute the payoffs of all players and show that they are maximal for the strategy profile stated in Theorem 4.1.

We start with the following lemma.

Lemma 4.2. Let $x, y \in\{0,1\}^{d}$ be two adjacent vertices in $H_{d}, d \geq 1$, and let $\left(p_{1}, \ldots, p_{4}\right)$ be a strategy profile with $\{x, y, \bar{y}\} \subseteq\left\{p_{1}, \ldots, p_{4}\right\}$ and $p_{i} \neq p_{j}$ for all $i, j \in[4], i \neq j$. Let $v \in\{0,1\}^{d}$ be a vertex and let $\delta:=\min _{i=1, \ldots, 4} \Delta\left(p_{i}, v\right)$.

If there exist $i, j \in[4], i \neq j$, such that $\Delta\left(p_{i}, v\right)=\Delta\left(p_{j}, v\right)=\delta$, then the vertex $v$ will not be colored by any player at the end of the propagation process.

Proof. Because the order of the players does not matter, we assume that $p_{1}=p \in\{0,1\}^{d}$, $p_{2}=x, p_{3}=y$, and $p_{4}=\bar{y}$. For the case $\delta \leq 1$, the statement clearly holds by definition of the propagation process. Hence, we consider $\delta \geq 2$. Note that for any two vertices $p_{i} \neq p_{j}$ with $\Delta\left(p_{i}, v\right)=\Delta\left(p_{j}, v\right)=\delta$, the distance $\Delta\left(p_{i}, p_{j}\right)$ must be even (and thus, at least two) because two vertices with an odd distance cannot have the same distance to any other vertex. Hence, $x$ and $y$ can never have the same distance $\delta$ to $v$ because their distance is one (they are adjacent). It follows that either two or three players have the same shortest distance $\delta$ to $v$ among all players. For both cases, we show that there always exist two neighbors of $v$, each having a different player with a unique closest distance of $\delta-1$. Using Observation 3.3, we can then conclude that these neighbors are colored in different colors and that $v$ is, thus, removed.

First, assume that exactly the two players $i$ and $j$ have distance $\delta$ to $v$, while the other two players have a distance larger than $\delta$. Then, $p_{i}$ and $p_{j}$ differ in $\Delta\left(p_{i}, p_{j}\right)=2 c$ bits for some $c \geq 1$. Note that $v$ equals $p_{i}$ in exactly $c$ of these bits and equals $p_{j}$ in the other $c$ bits (otherwise they cannot have the same distance to $v$ ). Hence, by swapping one of the $c$ bits where $v$ equals $p_{j}$, we reach a neighbor $u$ of $v$ such that $\Delta\left(p_{i}, u\right)=\delta-1<$ $\Delta\left(p_{j}, u\right)=\delta+1$. Analogously, swapping one of the $c$ bits where $v$ equals $p_{i}$ yields a neighbor $w$ with $\Delta\left(p_{j}, w\right)=\delta-1<\Delta\left(p_{i}, w\right)=\delta+1$. Note that the other two players have distance at least $\delta$ to both $u$ and $w$ since their distance to $v$ is at least $\delta+1$. Thus, $p_{i}$ has the unique shortest distance to $u$, and $p_{j}$ has the unique shortest distance to $w$. According to Observation 3.3, $u$ and $w$ are thus colored in different colors at time $\delta-1$. Consequently, $v$ is removed at time $\delta$.

Now, assume that exactly three players have the minimum distance $\delta$ to $v$. Since $x$ and $y$ are adjacent, we know that the only two possible cases are that $p, \bar{y}$, and either $x$ or $y$ have distance $\delta$ to $v$.

Case 1: $\Delta(p, v)=\Delta(x, v)=\Delta(\bar{y}, v)=\delta<\Delta(y, v)$. Note that $\Delta(y, v)=d-\Delta(\bar{y}, v)$, which yields $\delta<d / 2$. Note further that $x$ and $\bar{y}$ differ in an even number of $\Delta(x, \bar{y})=d-1$ bits, which means $d$ is odd. Hence, $\delta \leq(d-1) / 2$. Moreover, $v$ 
equals each of $x$ and $\bar{y}$ in exactly half of their $d-1$ differing bits (otherwise $v$ cannot have the same distance to both). It follows that $\delta=(d-1) / 2$ and it also holds that $x_{i}=\bar{y}_{i} \neq v_{i}$ is not possible for any $i \in[d]$ (this holds since $x_{i}=\bar{y}$ implied $\bar{y}=v_{i}$, which holds since $\left.\delta=(d-1) / 2\right)$. Now consider the vertex $p$ which also differs in $\delta$ bits to $v$. Clearly, $p$ and $v$ can neither differ in the same $\delta$ bits as $v$ and $x$, nor in the same $\delta$ bits as $v$ and $\bar{y}$ since then $p_{1}$ would be equal to either $x$ or $\bar{y}$. Hence, there exist indices $i$ and $j$ among the $d-1$ differing bits of $x$ and $\bar{y}$ such that $v_{i}=\bar{y}_{i}=p_{i} \neq x_{i}$ and $v_{j}=x_{j}=p_{j} \neq \bar{y}_{j}$. Thus, by swapping the $i$ th bit of $v$, we reach a neighbor $u$ with $\Delta(x, u)=\delta-1<\Delta(p, u)=\Delta(\bar{y}, u)=\delta+1$. Similarly, swapping the $j$ th bit of $v$ yields a neighbor $w$ with $\Delta(\bar{y}, w)=\delta-1<$ $\Delta(p, w)=\Delta(x, w)=\delta+1$. Clearly, also $y$ has distance at least $\delta$ to both $u$ and $w$. Hence, by Observation 3.3, $u$ and $w$ are colored in different colors and, thus, $v$ is removed.

Case 2: $\Delta(p, v)=\Delta(y, v)=\Delta(\bar{y}, v)=\delta<\Delta(x, v)$. Note that $\Delta(y, \bar{y})=d$ is even and that $v$ equals both $y$ and $\bar{y}$ in exactly $\delta=d / 2$ bits. As in Case 1 , the vertex $p$ cannot differ from $v$ in the same $\delta$ bits as $y$ or $\bar{y}$. Thus, we again find indices $i$ and $j$ with $v_{i}=\bar{y}_{i}=p_{i} \neq y_{i}$ and $v_{j}=y_{j}=p_{j} \neq \bar{y}_{j}$ such that for the corresponding neighbors $u$ and $w$ of $v$ we have $\Delta(y, u)=\delta-1<\Delta(p, u)=\Delta(\bar{y}, u)=\delta+1$ and $\Delta(\bar{y}, w)=\delta-1<\Delta(p, w)=\Delta(y, w)=\delta+1$. Since, by assumption, $x$ has distance at least $\delta$ from $u$ and $w$, it follows that $u$ and $w$ are colored in different colors and $v$ is removed.

Lemma 4.2 (together with Observation 3.3) shows that in every strategy profile as described in Theorem 4.1, the payoff of each player equals the number of vertices to which he/she has the unique minimum distance among all players. a profile.

The following lemma gives upper bounds on the possible payoffs for players in such

Lemma 4.3. Let $x, y \in\{0,1\}^{d}, d \geq 1$, and let $V_{x}^{y \bar{y}}:=\left\{v \in\{0,1\}^{d} \mid \Delta(v, x)<\right.$ $\min \{\Delta(v, y), \Delta(v, \bar{y})\}$. Then, $\left|V_{x}^{y \bar{y}}\right| \leq 2^{d-2}$. Moreoever, if $d$ is odd, then the bound is even smaller, that is,

$$
\left|V_{x}^{y \bar{y}}\right| \leq 2^{d-2}-\frac{1}{2}\left(\begin{array}{c}
d-1 \\
(d-1) / 2
\end{array}\right)
$$

Proof. Let $\alpha:=\Delta(x, y)$; thus, $\Delta(x, \bar{y})=d-\alpha$. For any vertex $v \in V_{x}^{y \bar{y}}$, it holds $\Delta(x, v)<\Delta(y, v)$ and also $\Delta(x, v)<\Delta(\bar{y}, v)$. The first inequality implies that $v$ equals $x$ in more than half of the $\alpha$ bits where $x$ and $y$ differ. Analogously, the second inequality implies that $v$ equals $x$ in more than half of the $d-\alpha$ bits where $x$ and $y$ differ. Clearly, the set of bits where $x$ and $y$ differ is disjoint from the set of bits where $x$ and $\bar{y}$ differ. Hence, the number of possible vertices in $V_{x}^{y \bar{y}}$ is

$$
\begin{aligned}
\left|V_{x}^{y \bar{y}}\right| & =\left(\sum_{\ell=\lceil(\alpha+1) / 2\rceil}^{\alpha}\left(\begin{array}{l}
\alpha \\
\ell
\end{array}\right)\right) \cdot\left(\sum_{\ell=\lceil(d-\alpha+1) / 2\rceil}^{d-\alpha}\left(\begin{array}{c}
d-\alpha \\
\ell
\end{array}\right)\right) \\
& \leq 2^{\alpha-1} \cdot 2^{d-\alpha-1}=2^{d-2},
\end{aligned}
$$


which proves the general bound. Now, for $d$ being odd, note that either $\alpha$ or $d-\alpha$ is even. We assume w.1.o.g. that $\alpha \leq d-1$ is even (that is, $d-\alpha$ is odd). Then, Equation (1) can be written as

$$
\begin{aligned}
\left|V_{x}^{y \bar{y}}\right| & =\left(\sum_{\ell=\alpha / 2+1}^{\alpha}\left(\begin{array}{c}
\alpha \\
\ell
\end{array}\right)\right) \cdot\left(\sum_{\ell=\lceil(d-\alpha) / 2\rceil}^{d-\alpha}\left(\begin{array}{c}
d-\alpha \\
\ell
\end{array}\right)\right) \\
& \leq\left(2^{\alpha-1}-\frac{1}{2}\left(\begin{array}{c}
\alpha \\
\alpha / 2
\end{array}\right)\right) \cdot 2^{d-\alpha-1} \\
& =2^{d-2}-\left(2^{d-\alpha-2} \cdot\left(\begin{array}{c}
\alpha \\
\alpha / 2
\end{array}\right)\right) .
\end{aligned}
$$

We now use the following identity for the central binomial coefficient

$$
\left(\begin{array}{c}
\alpha \\
\alpha / 2
\end{array}\right)=\frac{\alpha !}{((\alpha / 2) !)^{2}}=2^{\alpha} \cdot \frac{1 \cdot 3 \cdot \ldots \cdot(\alpha-1)}{2 \cdot 4 \cdot \ldots \cdot \alpha}
$$

to obtain

$$
\begin{aligned}
\left|V_{x}^{y \bar{y}}\right| & \leq 2^{d-2}-\left(2^{d-2} \cdot \frac{1 \cdot 3 \cdot \ldots \cdot(\alpha-1)}{2 \cdot 4 \cdot \ldots \cdot \alpha}\right) \\
& \leq 2^{d-2}-\left(2^{d-2} \cdot \frac{1 \cdot 3 \cdot \ldots \cdot(d-2)}{2 \cdot 4 \cdot \ldots \cdot(d-1)}\right) \\
& \leq 2^{d-2}-\frac{1}{2}\left(\begin{array}{c}
d-1 \\
(d-1) / 2
\end{array}\right) .
\end{aligned}
$$

We can now proceed with proving Theorem 4.1.

Proof of Theorem 4.1 To start with, observe that for $d=1$, the statement clearly holds since there are only two vertices in $H_{1}$, which gives a payoff of zero for each player, and it is not possible to obtain more than zero vertices for any player (by definition of the diffusion game).

In the following, we consider $d \geq 2$. Because the ordering of the players does not matter, we fix the strategy profile $\left(p_{1}=x, p_{2}=\bar{x}, p_{3}=y, p_{4}=\bar{y}\right)$. Moreover, due to the symmetry of the hypercube, we have to consider only the case that Player 1 changes her strategy. Let us first determine the payoff of Player 1 for the above profile. According to Observation 3.3 and Lemma 4.2, we know that Player 1 obtains exactly those vertices $v \in\{0,1\}^{d}$ to which he/she has the unique minimum distance: $\Delta(x, v)<\min \{\Delta(\bar{x}, v), \Delta(y, v), \Delta(\bar{y}, v)\}$. Recall that $x$ and $y$ are adjacent; that is, they differ in $\Delta(x, y)=1$ bit. Therefore, $\Delta(x, v)<$ $\Delta(y, v)$ implies that $v$ equals $x$ in that bit. Thus, we have $\Delta(y, v)=\Delta(x, v)+1, \Delta(\bar{x}, v)=$ $d-\Delta(x, v)$, and $\Delta(\bar{y}, v)=d-1-\Delta(x, v)$. Hence, $v$ has to satisfy $\Delta(x, v)<(d-1) / 2$ in order to satisfy $\Delta(x, v)<\Delta(\bar{y}, v)$ and $\Delta(x, v)<\Delta(\bar{x}, v)$. That is, $v$ is allowed to differ from $x$ in at most $\lfloor(d-1) / 2\rfloor$ of the $d-1$ bits where $x$ is equal to $y$. The payoff of Player 1 is, thus,

$$
\sum_{\ell=0}^{\lfloor(d-1) / 2\rfloor}\left(\begin{array}{c}
d-1 \\
\ell
\end{array}\right) .
$$

Note that this payoff equals $2^{d-2}$ if $d$ is even, and $2^{d-2}-\frac{1}{2}\left(\begin{array}{c}d-1 \\ (d-1) / 2\end{array}\right)$ if $d$ is odd. 
Now, let Player 1 choose an arbitrary vertex $p_{1} \in\{0,1\}^{d}$. Clearly, we can assume that $p_{1} \notin\{\bar{x}, y, \bar{y}\}$ since the payoff is zero otherwise. Hence, by Observation 3.3 and Lemma 4.2, we know again that the payoff of Player 1 equals the number of vertices in $\left\{v \in\{0,1\}^{d} \mid \Delta(x, v)<\min \{\Delta(\bar{x}, v), \Delta(y, v), \Delta(\bar{y}, v)\}\right\}$. By Lemma 4.3, we know that this number is at most $2^{d-2}$ if $d$ is even, and at most $2^{d-2}-\frac{1}{2}\left(\begin{array}{c}d-1 \\ (d-1) / 2\end{array}\right)$ if $d$ is odd. Therefore, Player 1 cannot increase the payoff by changing strategies, which finishes the proof.

The existence of Nash equilibria on hypercubes for three players as well as for more than four players remains open, in general.

\section{GENERAL GRAPHS}

In this section, we study the existence of Nash equilibria on arbitrary graphs. Using computer simulations, we found that for two players, a Nash equilibrium exists on any graph with at most $n=7$ vertices. For $n=8$, we obtained the graph depicted in Figure 6, for which there is no Nash equilibrium for two players. Because it is clear that adding isolated vertices to the graph in Figure 6 does not allow for a Nash equilibrium, we conclude the following.

Corollary 5.1. For two players, there is a Nash equilibrium on each $n$-vertex graph if and only if $n \leq 7$.

For more than two players, we can show the following.

Theorem 5.2. For any $k>2$ and any $n \geq\left\lfloor\frac{3}{2} k\right\rfloor+2$, there exists a tree with $n$ vertices such that there is no Nash equilibrium for $k$ players.

Proof. We describe a construction only for $n=\left\lfloor\frac{3}{2} k\right\rfloor+2$, because we can add arbitrarily many isolated vertices without introducing a Nash equilibrium.

We first describe the construction for $k$ being odd. We create one $P_{3}$, whose vertices we denote by $u_{1}, u_{2}$, and $u_{3}$, such that $u_{2}$ is the middle vertex of this $P_{3}$. For each $i \in\left[2,\left\lceil\frac{k}{2}\right\rceil\right]$, we create a copy of $P_{3}$, denoted by $P_{i}$, whose vertices we denote by $v_{i, 1}, v_{i, 2}$, and $v_{i, 3}$, such that $v_{i, 2}$ is the middle vertex of $P_{i}$. For each $i \in\left[2,\left\lceil\frac{k}{2}\right\rceil\right]$, we connect $v_{i, 1}$ to $u_{3}$. An example for $k=9$ is depicted in Figure 7.

To see that there is no Nash equilibrium for the constructed graph, consider first strategy profiles for which $u_{3}$ is free (that is, no player chooses $u_{3}$ ). If both $u_{1}$ and $u_{2}$ are

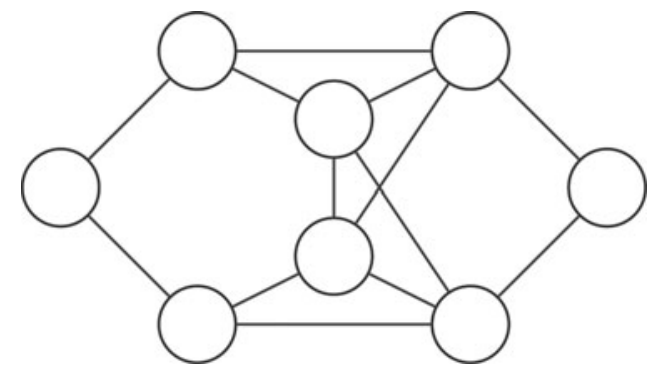

Figure 6 A graph on 8 vertices with no Nash equilibrium for two players. 


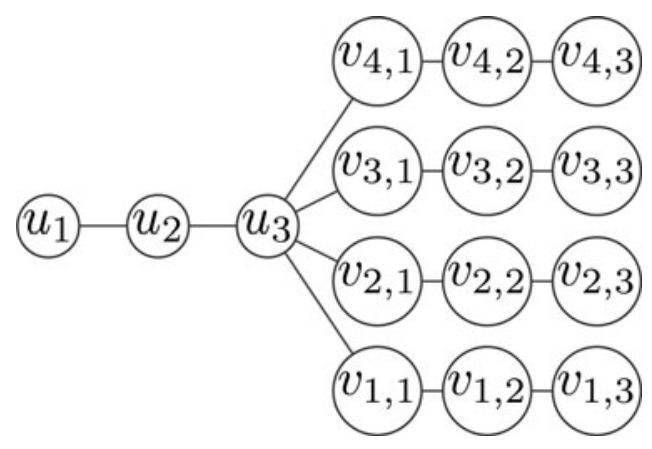

Figure 7 A tree with no Nash equilibrium, for 9 players.

free also, then there exists some $P_{i}$ with at least two occupied vertices (by the pigeon-hole principle). It is clear that at least one of the players occupying these vertices can increase the payoff by moving to $u_{3}$. If $u_{1}$ and $u_{2}$ are both occupied, then there exists some $P_{i}$ with at most one occupied vertex (again, by the pigeon-hole principle). It is clear that the player occupying this vertex can increase the payoff by moving to $u_{3}$. If only one vertex out of $u_{1}$ and $u_{2}$ is occupied, then the player occupying this vertex can increase the payoff by moving to $u_{3}$. Therefore, we can assume that $u_{3}$ is occupied. In this case, it holds that if both $u_{1}$ and $u_{2}$ are free, then at least one player has a payoff of 1 and gains more by moving to $u_{2}$. If both $u_{1}$ and $u_{2}$ are occupied, then at least one $P_{i}$ has at most one occupied vertex, and this occupied vertex can only be $v_{i, 1}$, therefore, the player occupying $u_{2}$ gains more by moving to $v_{i, 2}$. Finally, if exactly one out of $u_{1}$ and $u_{2}$ is free, then at least one $P_{i_{1}}$ has at most one occupied vertex, and this occupied vertex can only be $v_{i_{1}, 1}$. Moreover, at least one $P_{i_{2}}$ has at least two occupied vertices, therefore, a player occupying one of these vertices gains more by moving to $v_{i_{1}, 2}$. Therefore, this graph has no Nash equilibrium for $k$ players.

For $k$ being even, we create one $P_{2}$, whose vertices we denote by $u_{1}, u_{2}$. For each $i \in\left[2, \frac{k}{2}+1\right]$, we create a copy of $P_{3}$, denoted by $P_{i}$, whose vertices we denote by $v_{i, 1}$, $v_{i, 2}$, and $v_{i, 3}$, such that $v_{i, 2}$ is the middle vertex of $P_{i}$. For each $i \in\left[2, \frac{k}{2}+1\right]$, we connect $v_{i, 1}$ to $u_{2}$. This graph has no Nash equilibrium for $k$ players, as can be verified by a similar analysis as above.

\section{CONCLUSION}

We studied a competitive diffusion game for three or more players on several classes of graphs, answering — as a main contribution — an open question concerning the existence of a Nash equilibrium for three players on grids [8] negatively. Further, extending previous results on hypercubes [3], we proved that Nash equilibria always exist for four players on $d$-dimensional hypercubes. With this work, we provide a first systematic study of this game for more than two players. However, there are several questions left open, of which we mention some here.

An immediate question (generalizing Theorem 3.1) is whether a Nash equilibrium exists for more than three players on a grid. Computer simulations lead us to conjecture that there is no Nash equilibrium for four players on a grid of size larger than $6 \times 6$. A further immediate question (generalizing Theorem 4.1) is whether a Nash equilibrium exists for three players or more than four players on a $d$-dimensional hypercube. Also, giving a lower 
bound for the number of vertices $n$ such that there is a graph with $n$ vertices with no Nash equilibrium for $k$ players is an interesting question because it is not clear that the upper bounds given in Theorem 5.2 are optimal. In other words, is it true that $n \leq \frac{3}{2} k+1$ implies the existence of a Nash equilibrium for $k$ players?

\section{ACKNOWLEDGMENT}

We thank Manuela Hopp for helpful discussions and implementations for the result on hypercubes.

\section{FUNDING}

Laurent Bulteau was supported by the Alexander von Humboldt Foundation, Bonn, Germany. Main work done while affiliated with TU Berlin. Vincent Froese was supported by the DFG, project DAMM (NI 369/13). Nimrod Talmon was supported by DFG Research Training Group Methods for Discrete Structures (GRK 1408). Main work done while affiliated with TU Berlin.

\section{REFERENCES}

[1] N. Alon, M. Feldman, A. D. Procaccia, and M. Tennenholtz. "A Note on Competitive Diffusion through Social Networks.” Information Processing Letters 110:6 (2010), 221-225.

[2] C. Dürr and N. K. Thang. "Nash Equilibria in Voronoi Games on Graphs." In Proceedings of the 15th Annual European Symposium on Algorithms (ESA'07), pp. 17-28. LNCS 4698, Springer, 2007.

[3] S. R. Etesami and T. Basar. "Complexity of Equilibrium in Diffusion Games on Social Networks.” Automatica, 68 (2016), 100-110.

[4] T. Ito, Y. Otachi, T. Saitoh, H. Satoh, K. Yamanaka, and X. Zhou. "Competitive Diffusion on Weighted Graphs." In Proceedings of the 14th International Symposium on Algorithms and Data Structures (WADS '15), p. 422, LNCS 9214. Springer, 2015.

[5] J. Janssen and C. Vautour. "Finding Safe Strategies for Competitive Diffusion on Trees." Internet Mathematics 11:3 (2015), 232-252.

[6] D. Kempe, J. Kleinberg, and É. Tardos. "Maximizing the Spread of Influence through a Social Network." In Proceedings of the Ninth ACM SIGKDD International Conference on Knowledge Discovery and Data Mining, pp. 137-146. New York, NY: ACM, 2003.

[7] M. Mavronicolas, B. Monien, V. G. Papadopoulou, and F. Schoppmann. "Voronoi Games on Cycle Graphs." In Proceedings of the 33rd International Symposium on Mathematical Foundations of Computer Science (MFCS'08), pp. 503-514, LNCS 5162. Springer, 2008.

[8] E. Roshanbin. "The Competitive Diffusion Game in Classes of Graphs." In Proceedings of the 10th International Conference on Algorithmic Aspects in Information and Management (AAIM'14), pp. 275-287, LNCS 8546. Springer, 2014.

[9] L. Small. "Information Diffusion on Social Networks." PhD thesis, National University of Ireland Maynooth, 2012.

[10] L. Small and O. Mason. "Nash Equilibria for Competitive Information Diffusion on Trees." Information Processing Letters 113:7 (2013), 217-219.

[11] R. Takehara, M. Hachimori, and M. Shigeno. "A Comment on Pure-Strategy Nash Equilibria in Competitive Diffusion Games." Information Processing Letters 112:3 (2012), 59-60.

[12] V. Tzoumas, C. Amanatidis, and E. Markakis. "A Game-Theoretic Analysis of a Competitive Diffusion Process over Social Networks." In Proceedings of the Eighth Workshop on Internet and Network Economics (WINE’12), pp. 1-14, LNCS 7695. Springer, 2012. 